\title{
Clustered Standard Deviation and Its Benefit to Identify Atrial Fibrillation
}

\author{
Filip Plesinger ${ }^{1}$, Ivo Viscor ${ }^{1}$, Petr Nejedly ${ }^{1}$, Veronika Bulkova ${ }^{2}$, Josef Halamek ${ }^{1}$, Pavel Jurak ${ }^{1}$ \\ ${ }^{1}$ Institute of Scientific Instruments of the CAS, Brno, Czech Republic \\ ${ }^{2}$ Medical Data Transfer, Brno, Czech Republic
}

\begin{abstract}
Background: Atrial fibrillation $(A F)$ is a dysfunction of heart atriums shown as irregular heart activity leading to a higher risk of heart failure. Since AF may occur episodically, it is usually diagnosed using ECG Holter recordings. However, the presence of other pathologies and noise makes the automated processing of ECG Holter recordings complicated. Here, we present a new feature to distinguish AF from sinus rhythm as well as from other pathologies: Clustered Standard Deviation $\left(C_{S T D}\right)$.

Method: QRS complexes are extracted from the ECG signal, and inter-beat intervals $(R R)$ are ordered by their length. Then, RR clusters are found and the mean $R R$ value is computed for each RR cluster. $C_{S T D}$ is computed using a formula for standard deviation using cluster-specific mean values instead of a global mean.

Results: $C_{S T D}$ was evaluated for 7,254 ECG segments from a private dataset (MDT company, Brno, Czechia), 60 seconds length, 1-lead, $250 \mathrm{~Hz}$ sampling frequency. $C_{S T D}$ showed high values for $A F$ while remaining low for other pathologies and sinus rhythm. $C_{S T D}$ between $A F$ and other classes showed AUC 0.95. For comparison, a standard deviation of RR intervals leads to AUC 0.65 due to its sensitivity to other pathologies. Test on public MIT-AFDB dataset shown AUC and AUPRC 0.98 and 0.97, respectively.
\end{abstract}

\section{Introduction}

The Atrial Fibrillation (AF) causes an irregular heart rhythm leading to a random distribution of QRS complexes. Regular P-wave is not present; instead, socalled f-waves are present in the whole ECG signal. In the past, several approaches to detect atrial fibrillation has been proposed, ranging from analysis of ventricular response [1]-[3] to more complex signal analysis [4] implementing machine-learning techniques including convolutional neural networks [5], [6]. Although this description might lead to the impression that detecting $\mathrm{AF}$ is already solved the issue, the CinC/Physionet Challenge 2017 [7] shown that this task is still challenging, especially under specific circumstances. Those specific circumstances mean cases when other pathologies must be expected in the ECG signal and/or the signal is collected from ECG holters during usual daily activities of patients; the usual daily activities result in a larger amount of noise caused by movement and its technical consequences as poor or broken contacts.

In this paper, we present novel descriptor sensitive to $\mathrm{AF}$ in one-lead ECG holter recordings.

\section{Method}

Here we present Clustered Standard Deviation ( $\mathrm{C}_{\text {STD }}$ ), which is a modification of standard deviation (STD). While STD works well for separation of AF and sinus rhythm (SR), it is insufficient when other pathologies are present (e.g., premature ventricular contractions - PVC in bigeminy linking).

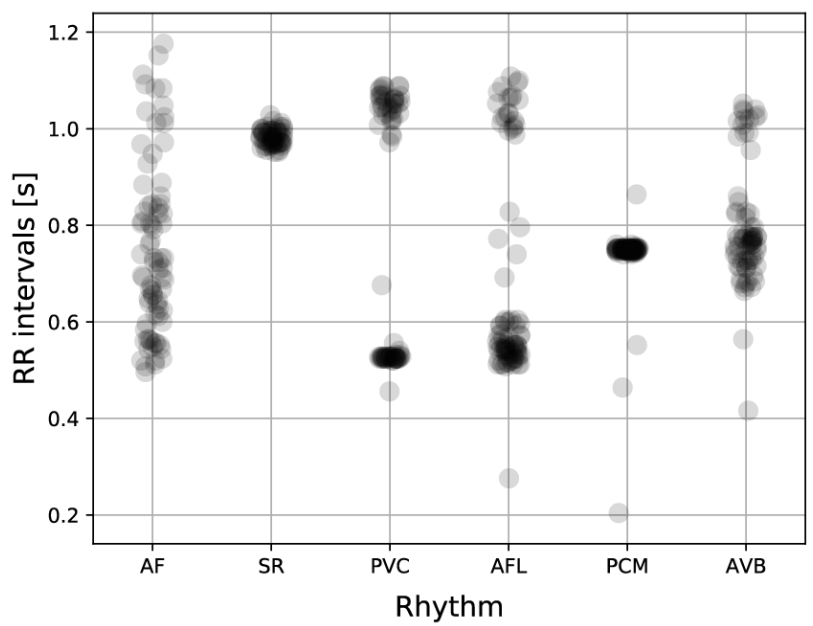

Figure 1. Examples of RR distributions in 60-second recordings of different rhythms. AF-Atrial Fibrillation, SR - sinus rhythm, PVC - premature ventricular contractions, AFL -atrial flutter, PCM - paced rhythm, AVB $-2^{\text {nd }}$ degree AV block 
Specific sign of AF is that $\mathrm{QRS}$ complexes are distributed in random; therefore, RR intervals do not produce clusters. Other pathologies, even if they show high STD, lead to some rhythm regularity - the mentioned PVC bigeminy will produce two clusters of RR intervals - short and long, as shown in Fig.1-PVC.

\subsection{Clustered Standard Deviation}

From the Fig. 1 follows that if the STD is computed concerning RR clusters, then AF should still produce high values because it will form one (or more) wide clusters (Fig1-AF). Other pathologies and sinus rhythm (Fig1-SR) should provide low values since they can be clusterized into one or more tight clusters. Therefore, clustering of RR intervals is the key to the presented approach. The whole computational process is designed as follows:

First, QRS complexes are detected using a method based on envelograms [8], and inter-beat (RR) intervals are extracted.

Next, RR intervals are sorted by their value. Whenever a gap between neighboring (sorted) RR intervals is bigger then a limit $L_{G}$, a new RR cluster is defined. The limit $L_{G}$ is computed as follows:

$$
L_{G}=\max ([a \times S T D(R R), b])
$$

Optimal values of $a$ and $b$ coefficients were found later using the grid-search method using the whole dataset.

When RR intervals are clustered, the average RR interval $A_{c}$ for each cluster $c$ is computed. Finally, the $C_{S T D}$ is computed as follows:

$$
C_{S T D}=\sqrt{\frac{1}{N} \sum_{i=1}^{N}\left(R R_{i}-A_{c i}\right)^{2}}
$$

where $N$ is the number of RR intervals, and the $A_{c i}$ is an average RR of the cluster to which specific $R R_{i}$ belongs.

\subsection{Finding coefficients and evaluation}

To observe $C_{S T D}$ behavior and to optimize limiting coefficients, we used private dataset (MDT company, Brno, Czech Republic). The dataset consisted of 123 onehour, one-lead ECG recordings sampled at $250 \mathrm{~Hz}$. These recordings were split into 7,254 non-overlapping segments of length 60 seconds. Rhythms present in this private dataset are described in Tab. 1
Table 1. Description of rhythms in the private dataset

\begin{tabular}{lr}
\hline Rhythm & ECG Segments \\
\hline Atrial fibrillation & 1,062 \\
Atrial flutter & 649 \\
Atrial tachycardia & 649 \\
AV-block & 236 \\
Premature atrial contractions & 590 \\
Premature ventricular contractions & 1,063 \\
Sinus rhythm & 2,121 \\
Noise & 472 \\
\hline
\end{tabular}

To maximize the $\mathrm{C}_{\mathrm{STD}}$ capability to isolate $\mathrm{AF}$, we needed to find proper values of limiting coefficients. Therefore, we used the grid-search approach and evaluated area under the curve (AUC) as well as area under the precision-recall curve (AUPRC). Since we found that $\mathrm{C}_{\text {STD }}$ can also be used to identify noisy recordings, we also run another grid search to find the best coefficients to isolate noisy recordings.

After the coefficients where optimized, we processed independent dataset MIT-AFDB [1] from PhysioNET [9] and evaluated the same AUC and AUPRC metrics. From the original 25 files, we excluded two due to missing data; finally, we extracted 13, 380 recodings (60 seconds long, containing only a single rhythm) using the WFDB-Python package [10]; the recordings extracted from the dataset are shown in Tab. 2

Table 2. Description of rhythms in the AFDB dataset

\begin{tabular}{lr}
\hline Rhythm & ECG segments \\
\hline Atrial fibrillation & 5,289 \\
Atrial flutter & 86 \\
Sinus rhythm & 8,005 \\
\hline
\end{tabular}

\section{Results}

The grid search (to isolate $\mathrm{AF}$ ) found limiting coefficients $a=0.05$ [-] and $b=0.06 \mathrm{~s}$. AUC and AUPRC on the private dataset shown 0.95 and 0.76 , respectively. $\mathrm{C}_{\text {STD }}$ values for different rhythm are shown in Fig.2-top. Evaluation using the public AFDB dataset shown AUC and AUPRC 0.98 and 0.97, respectively.

For comparison purposes, we also evaluated a standard deviation (STD) of RR intervals (Fig2-bottom). Using STD shown AUC and AUPRC of 0.73 and 0.23 , respectively. 

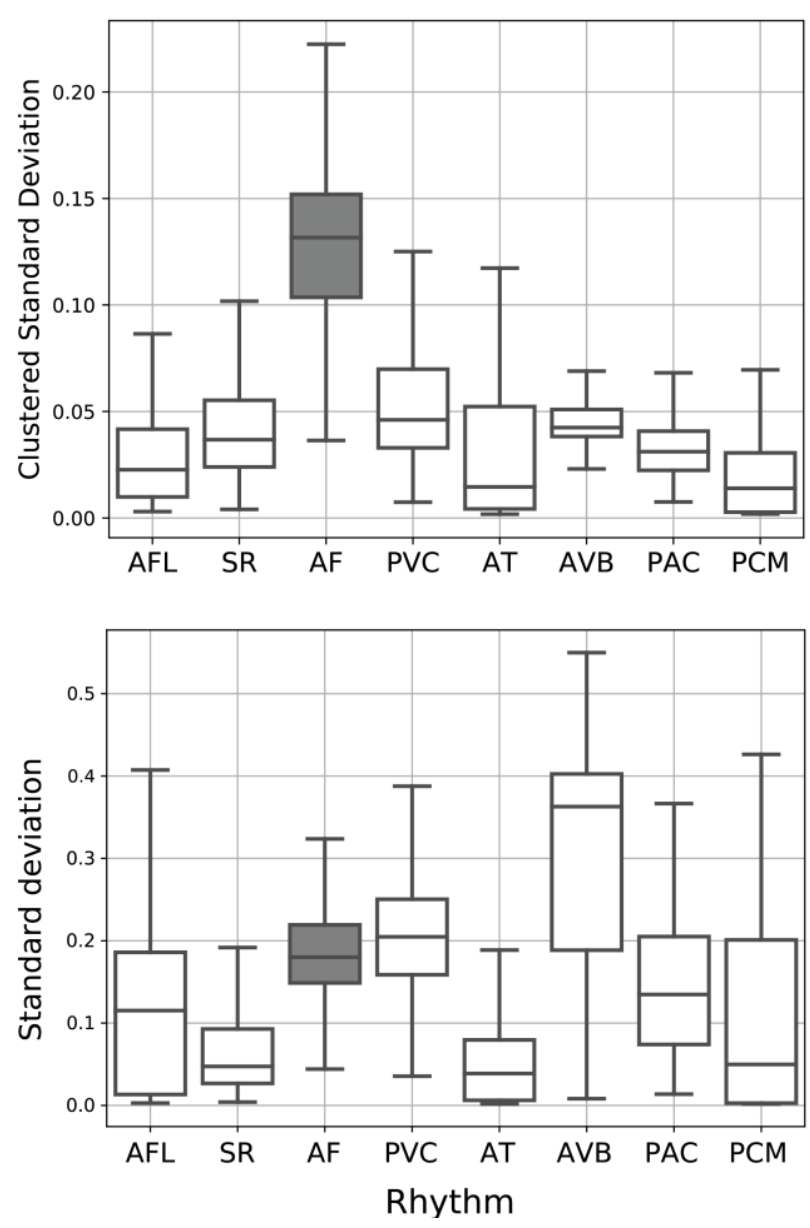

Figure 2. Comparison of the Clustered Standard Deviation (top) and the Standard Deviation (bottom) for different rhythms. Clustering limits were set to $a=0.05$ and $b=0.06$. Private dataset (MDT, Brno, CZ) consisting of 7, 254 ECG segments was used. AFL-Atrial flutter, SR - sinus rhythm, AF-Atrial Fibrillation (gray), PVC - premature ventricular contractions, $A T-$ sinus tachycardia, $\mathrm{AVB}-2^{\text {nd }}$ degree AV block, PAC - premature atrial contractions, PCM paced rhythm.

Another grid-search run to isolate noisy recordings shown $a=6[-]$ and $b=0.04 \mathrm{~s}$. This setting of $\mathrm{C}_{\mathrm{STD}}$ coefficients shown a strong ability to isolate noisy recordings with AUC and AUPRC 0.997 and 0.974, respectively (Fig.3).

A comparison of $\mathrm{C}_{\mathrm{STD}}$ for $\mathrm{AF}$ versus sinus rhythm is shown in Fig. 4 for both the private and public datasets.

We also tested the $\mathrm{C}_{\text {STD }}$ for different sizes of input $\mathrm{ECG}$ signal varying from 5 to 60 seconds; the one-second step was used. Fig.5 shows method performance using the private dataset on shortened ECG segments. Limiting coefficients were set to $a=0.05$ and $b=0.06$.

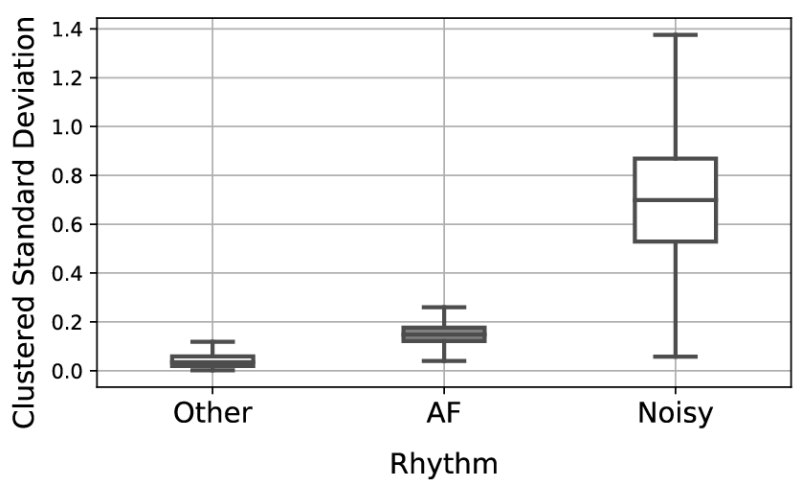

Figure 3. Clustered Standard Deviation could be used to detect noisy recordings if different clustering limits $(\mathrm{a}=6$ and $b=0.04)$ are applied.

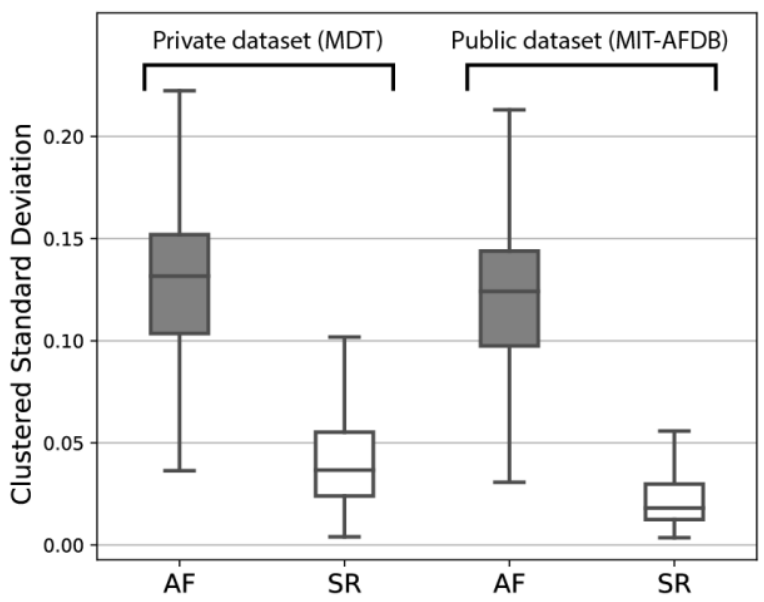

Figure 4. $\mathrm{C}_{\mathrm{STD}}$ for atrial fibrillation (AF) and sinus rhythm (SR) for private (MDT) and public (AFDB) datasets

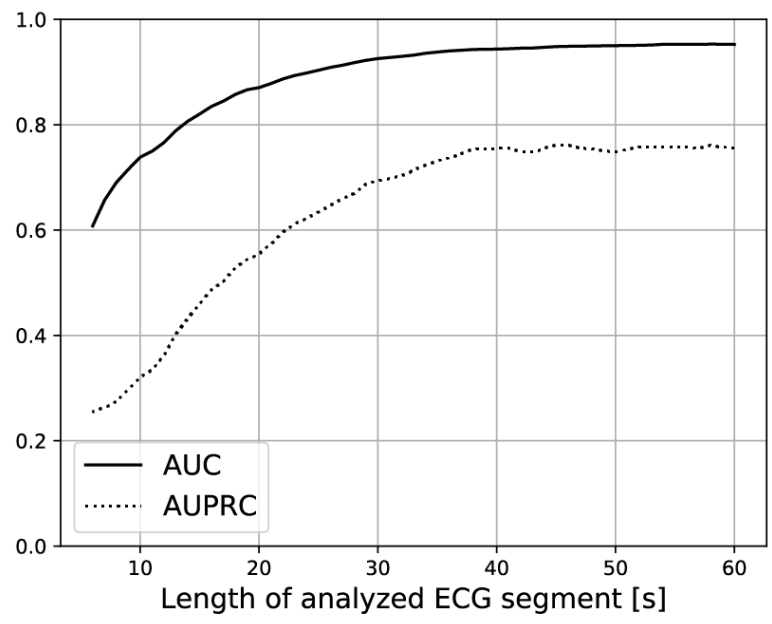

Figure 5. Method performance by the length of source ECG segment. AUC-area under the curve, AUPRC - area under the precision-recall curve. The performance was tested using 7,254 ECG segments from the private dataset. 


\section{Discussion}

Presented results showed that the $\mathrm{C}_{\mathrm{STD}}$ has strong potential for AF detection; and, furthermore, that the $\mathrm{C}_{\mathrm{STD}}$ can separate AF from other pathologies as well. On the other hand, Fig.2 showed that even the best possible threshold for $\mathrm{C}_{\mathrm{STD}}$ would result in some amount of false positives and negatives. Therefore, $\mathrm{C}_{\mathrm{STD}}$ (for $\mathrm{AF}$ detection) should not be used alone, but in combination with other features as [11]. However, for comparison purposes we found a $\mathrm{C}_{\mathrm{STD}}$ threshold at 0.081 , producing the best $\mathrm{F} 1$ score for the private dataset. Then we computed the F1 score on the AFDB dataset using the same threshold and compared it with existing methods (Tab.3). The performance of the $\mathrm{C}_{\mathrm{STD}}$ is comparable to less-complex models. The presented approach was not affected by the AFDB data; however, threshold retrained for AFDB would lead to F1 score of 0.93 .

Table 3. Comparison to other methods - AFDB dataset.

\begin{tabular}{lr}
\hline Approach & F1 score \\
\hline Corrected conditional Entropy [12] & 0.85 \\
This paper-CSTD & 0.89 \\
Markov model [1] & 0.90 \\
Convolutional neural networks [6] & 0.99 \\
\hline
\end{tabular}

Tests using different lengths of ECG segments (Fig. 5) reveals that the usability of $\mathrm{C}_{\mathrm{STD}}$ decreases if shorter segments then 40 seconds are used, but further elaborating with limiting coefficients could help to reduce this loss.

In addition to our primary goal, results in figure 3 suggested that $\mathrm{C}_{\text {STD }}$ could be successfully used for the detection of noisy recordings, but it should be mentioned that these results will probably change when different QRS detection method is used (for example a method less sensitive to noise artifacts).

Finally, results from the independent AFDB dataset (Fig.4) shown a similar trend in $\mathrm{C}_{\mathrm{STD}}$; in SR from AFDB $\mathrm{C}_{\text {STD }}$ shown smaller variation probably reflecting the fact that AFDB files were recorded in the resting supine position while private MDT dataset was recorded during a common daily activity of patients.

\section{Conclusion}

We presented a novel feature to detect AF, the Clustered Standard Deviation. Using the private dataset we have shown that the feature is valuable especially if other rhythm disturbances are expected, which is a common case in holter ECG processing. Test on public AFDB dataset showed that $\mathrm{C}_{\mathrm{STD}}$ works in the same manner with the independent data. In addition, we also showed that the presented method could be used to detect noisy recordings if different limiting coefficients are used.

\section{Acknowledgments}

This work was supported by project TG03010046-10 by the Czech Technological Agency and by the Ministry of Education, Youth and Sports of the Czech Republic (project LO1212).

\section{References}

[1] G. B. Moody and R. G. Mark, "A New Method for Detecting Atrial Fibrillation Using RR Intervals," Computers in Cardiology. pp. 227-230, 1983.

[2] C. Huang, S. Ye, H. Chen, D. Li, F. He, and Y. Tu, "A novel method for detection of the transition between atrial fibrillation and sinus rhythm," IEEE Trans. Biomed. Eng., 2011.

[3] I. Christov, V. Krasteva, I. Simova, T. Neycheva, and R. Schmid, "Multi-parametric analysis for atrial fibrillation classification in ECG," in Computing in Cardiology, 2017.

[4] C. Hoog Antink, S. Leonhardt, and M. Walter, "Fusing QRS Detection, Waveform Features, and Robust Interval Estimation with a Random Forest to Classify Atrial Fibrillation," in Comput Cardiol (Rennes IEEE), 2017, vol. 44, pp. 1-4.

[5] Y. Xia, N. Wulan, K. Wang, and H. Zhang, "Detecting atrial fibrillation by deep convolutional neural networks," Comput. Biol. Med., vol. 93, no. July 2017, pp. 84-92, 2018.

[6] R. He et al., "Automatic detection of atrial fibrillation based on continuous wavelet transform and 2D convolutional neural networks," Front. Physiol., 2018.

[7] G. D. Clifford et al., "AF Classification from a Short Single Lead ECG Recording: The Physionet Computing in Cardiology Challenge 2017," in Comput Cardiol (Rennes IEEE), 2017, pp. 1-4.

[8] F. Plesinger, P. Nejedly, I. Viscor, J. Halamek, and P. Jurak, "Parallel use of a convolutional neural network and bagged tree ensemble for the classification of Holter ECG," Physiol. Meas., vol. 39, no. 9, 2018.

[9] A. L. Goldberger et al., "PhysioBank, PhysioToolkit, and PhysioNet: components of a new research resource for complex physiologic signals.," Circulation, vol. 101, no. 23, pp. E215-E220, 2000.

[10] I. Silva and G. B. Moody, "An Open-source Toolbox for Analysing and Processing PhysioNet Databases in MATLAB and Octave," J. Open Res. Softw., 2014.

[11] F. Plesinger, P. Andrla, I. Viscor, J. Halamek, V. Bulkova, and P. Jurak, "Shape Analysis of Consecutive Beats May Help in the Automated Detection of Atrial Fibrillation," in Computing in Cardiology (CinC), 2018, pp. 1-4.

[12] S. Cerutti, L. T. Mainardi, A. Porta, and A. M. Bianchi, "Analysis of the dynamics of RR interval series for the detection of Atrial Fibrillation episodes," in Computers in Cardiology, 1997.

Address for correspondence:

Filip Plesinger, Kralovopolska 147, Brno, Czech Republic fplesinger@isibrno.cz 